\title{
Lineamientos para la manufactura de partes de alta precisión
}

\author{
Guidelines for high precision manufacturing
}

Natalia Robles-Obando'

Fecha de recepción: 21 de enero del 2014

Fecha de aprobación: 26 de abril del 2014

Robles-Obando, N. Lineamientos para la manufactura de partes de alta precisión. Tecnología en Marcha. Vol. 27, Nº I. Pág 48-56 


\section{Palabras clave}

Manufactura de partes; alta precisión.

\section{Resumen}

La asignación de tolerancias para las dimensiones de las partes tiene un efecto importante tanto en el costo de la manufactura como en su calidad. Una manufactura muy precisa implica designar tolerancias estrictas para las dimensiones, lo que se traduce en un alto costo del producto. Actualmente, la filosofía de calidad consiste en buscar la excelencia. La investigación que se resume en este artículo tuvo como objetivo establecer los factores que influyen en la mejora de las prácticas de manufactura de partes de acoples o ensambles.

Estos factores resultaron ser el ajuste requerido para el acople final, los equipos y herramientas disponibles para el maquinado, el sistema de producción, los equipos y métodos de medición, las condiciones ambientales durante el proceso, el tamaño de las partes, los métodos de manipulación, así como el material utilizado y los procesos o usos posteriores. En la investigación también se estudió el impacto de la filosofía de calidad usada para la manufactura, encontrando que la exactitud de la máquina determina en gran medida la capacidad de cumplimiento de los valores dimensionales especificados.

\section{Key words}

Parts machining; high precision.

\begin{abstract}
Tolerances for dimensions have a great impact in manufacturing costs and quality. When tolerances for dimensions are strict the manufacturing costs increase. Today's quality philosophy concentrates on search for excellence. This research objective was to determine the factors that influence the improvement of manufacturing practices of assemblies. Among the factors found are the assembly allowance or fit, the machining equipment and tools, the production system, the measurement equipment and methods, the environmental conditions of the process, the parts size and manipulation methods, the material and future use and process of the parts. The quality philosophy also was considered, in fact, it showed to have a great impact. The machine accuracy also had a great impact in achieving final dimensions.
\end{abstract}




\section{Dimensiones y tolerancias}

Bjorke (1989) define dimensión como una dimensión geométrica de longitud o ángulo, que tiene un elemento de referencia que puede ser la superficie de una parte pero también un centro de línea o un plano de referencia. El diseñador de la parte es el que determina el elemento de referencia, basado en un análisis de los requerimientos funcionales de las partes. La zona de tolerancia para la dimensión está definida por su tamaño y su posición en relación con el tamaño básico de la dimensión. El tamaño de la tolerancia es la diferencia entre los límites máximo y mínimo para la dimensión. Los estándares de los fundamentos para el diseño de productos y procesos, así como de los dibujos técnicos, están definidos en ASMEY I 4.5M- 1994 y ASMEY I 4.5.2-2000GDTP. Fowlkes y Creveling (1995) indican que el tema de la geometría dimensional y tolerancia (GD\&T) es un proceso de comunicación de especificaciones que se enfoca en el desarrollo de un modelo gráfico del diseño después de que el modelo analítico y físico ha sido desarrollado a través de tolerancias.

Wortman (2006) define una tolerancia bilateral cuando se permite hacia ambos lados del elemento de referencia una desviación positiva para la dimensión de la parte. Maghsoodloo (2000) señala que se designan tolerancias unilaterales cuando las desviaciones del valor especificado se permiten solo para un extremo, ya sea solo una desviación mayor, con un límite permisible superior, o por el contrario solo una desviación inferior, con un límite permisible solo inferior.

Hay procesos de manufactura, como los de maquinado de roscas, que usan tolerancias unilaterales en los diseños de sus partes debido a que la variación ocurre en una dirección. Bengtson (2008) sugiere que este hecho se debe reconocer para poder calibrar el parámetro objetivo en el maquinado. Maghsoodloo (2000) también indica la existencia de tolerancias asimétricas para el maquinado, que son casos donde la tolerancia especificada es mayor hacia un extremo que hacia el otro, es decir, cuando no hay proporcionalidad. Las tolerancias, unilaterales o asimétricas, representan la situación de muchas piezas, donde la desviación hacia un lado del valor de la dimensión meta es más perjudicial que hacia otro.

Bjorke (1989) considera que es imposible la manufactura de partes exactamente iguales, y más allá tampoco es necesario. McMahon (1998), en sus guías para la simplificación y estandarización en el diseño de productos, estima que los diseñadores deben procurar evitar el uso de tolerancias ajustadas o acabados de superficie de alta calidad. Es por eso que Fowlkes y Creveling (1995) indican que designar el valor meta de la dimensión, así como el valor de sus tolerancias, implica balancear el costo del producto con la calidad.

Según Maghsoodloo (2000), ante tolerancias asimétricas, los diseñadores acostumbran reducirlas a un solo valor y asignarlas entonces bilateralmente, o bien colocar el valor meta en medio de ambas tolerancias. En los casos de elementos acoplados, lo primordial es lograr el ajuste especificado para el acople y es en función de éste como se establecen las tolerancias para las partes. Tanto las tolerancias como los ajustes los establecen los ingenieros bajo la consideración de la incertidumbre del diseño y de la producción.

Mehta (2010) también indica que la práctica usual en el maquinado de partes, cuyos diseños establecen tolerancias del tipo unilateral, es colocar como objetivo dimensional meta el valor central entre el objetivo y la tolerancia unilateral. Este mismo autor considera que en ocasiones se usan las tolerancias como si fueran límites permisibles para la especificación dimensional. Dicha práctica se realiza bajo el concepto de que todo lo que se encuentre entre el valor meta y el límite permitido cumple con las especificaciones y por ende no corresponde a un defecto, propio de la filosofía de Cero Defectos. Esta práctica confunde el sentido de la asignación de una tolerancia a una dimensión. Burton (20/2) apunta que las filosofías de mejoramiento actuales se basan en un concepto de búsqueda de la excelencia.

Fowlkes y Creveling (1995) afirman que las tolerancias que se asignan a las dimensiones impactan tanto el costo como la calidad de la manufactura y llegan a impactar incluso el nivel de satisfacción del cliente. Creveling (1997) sugiere contabilizar en el costo del ciclo de vida del producto los costos incurridos por las reparaciones o incluso la reposición del producto, que son los costos de un desempeño desviado del cumplimiento del objetivo, a pesar de estar dentro de la tolerancia permitida para la dimensión. La satisfacción del cliente se ve afectada al no cumplir la parte con las especificaciones. Creveling (1997) considera la función de pérdida de la calidad de Taguchi para la toma de decisiones respecto a las 
tolerancias. De esta manera, los esfuerzos de mejora deben estar orientados a reducir la variación entre el desempeño meta y el real, para que la pérdida sea cada vez más pequeña. La función pérdida de calidad de Taguchi contrasta con el pensamiento que solo penaliza cuando se está fuera de las especificaciones. Taguchi, en sus imperativos para la calidad, propone que las tolerancias se definan antes de la manufactura, pues de esta forma se podría determinar la función de pérdida de la calidad antes de la manufactura. Al-Ansary y Deiab (1997) presentan una investigación del balance de estos aspectos, buscando optimizar la designación de las tolerancias que impliquen cumplir con aspectos de calidad al menor costo posible.

La obtención de resultados más cercanos a los meta es una tarea que según Lin y otros (1997) solo puede darse cuando se ha conseguido cierto nivel de capacidad del proceso, pues solo cuando un proceso ha logrado tener capacidad, necesariamente mediante la inversión en aspectos como la planta, el equipo, la maquinaria o la capacitación al personal, es cuando podrá tener resultados más precisos o alta precisión en la manufactura. Un proceso se cataloga como capaz cuando su dispersión tiene forma de campana y su rango de variación es menor a lo que los límites de la especificación permiten para el producto.

\section{Metodología de la investigación}

Con base en los hallazgos de la investigación de las prácticas actuales de manufactura de partes, y según lo encontrado en la revisión de la literatura, se establecerán los factores o lineamientos por considerar para la mejora de las prácticas y posteriormente se presentarán en un modelo para guiar la manufactura bajo la consideración de estos.

\section{Factores por considerar en la manufactura de partes}

A continuación se presentan los factores que en la investigación se identificaron como importantes para la mejora de las prácticas de manufactura de partes de acoples o ensambles.

\section{I.Ajuste requerido para el acople final}

Los estándares de geometría dimensional y tolerancia (GT\&D) establecen cinco tipos básicos de ajustes. La American Society of Mechanical Engineers (ASME), en sus estándares, como el ASME Y |4-52009, presentan las tolerancias para especificaciones para distintos tipos de ajustes, por ejemplo holgado, medio, ajustado, etc.). Estos estándares son una herramienta para el diseño, pues permiten uniformidad en las especificaciones e interpretaciones y según la ASME reducen el trabajo de manufactura por esta claridad en el diseño. Existen otras normas para el diseño, como ISO 286, aunque las de la ASME son las preferidas.

Este detalle es fundamental, pues generalmente el ajuste final es el que determina su calidad. Los diseños usualmente se plasman en planos, que para el caso de los ensambles deben considerar la tolerancia del ensamble final y no solo el reporte aislado de cada pieza.

Un aspecto que se debe destacar respecto al ajuste de los acoples de piezas es que en la investigación se encontró la necesidad de mejorar el dominio de esta técnica para comprender a cabalidad el concepto de la tolerancia del ajuste y el traslado de esa tolerancia hacia las partes del acople.

\section{Equipos y herramientas para el maquinado}

La capacidad de maquinado está dada por la precisión de la herramienta, incluso más allá de si el maquinado es $\mathrm{CNC}$ o es en máquina convencional. En la investigación se encontró que si bien el maquinado CNC conlleva una serie de bondades, no es necesariamente determinante al hablar de lograr o no una dimensión final. La habilidad del operador es determinante siendo éste el que saca provecho de las herramientas disponibles.

Hay que destacar que el factor anterior está ligado al sistema de producción; ya sea un sistema por proceso o taller o un sistema de producción repetitivo. La maquinaria CNC requiere elaborar el programa para maquinar la parte, lo que es atractivo para altos volúmenes de producción pero no tanto para bajos.

\section{Equipos para la medición}

Los equipos de medición de dimensiones deben tener la capacidad de medir las especificaciones indicadas para poder garantizar que se revisará si se está logrando la precisión buscada. Este equipo requiere además un mantenimiento y calibración periódicos. Sin embargo, cabe destacar que el equipo calibrado 
que no permita determinar las mediciones requeridas igualmente no es apto para el proceso. Por tanto, en general se puede decir que el equipo debe tener la resolución adecuada. Este equipo puede implicar desde medidores de altura hasta calibradores especiales (gages) según la pieza y la precisión de la dimensión final que se busca. Este factor se debe revisar desde el diseño de la parte pues la dimensión meta que se requiere debe poder medirse.

\section{Métodos de medición}

Además del equipo de medición, entra en juego el método de medición, pues pequeños detalles, como sujetar con la mano una pieza aplicando alguna fuerza mínima, pueden alterar la dimensión meta aunque no sea más que por un par de minutos.

Además, los métodos que se siguen al realizar las mediciones se deben estandarizar entre los operadores, para reducir la probabilidad de cometer errores debido al método seguido o diferencias entre operadores.

\section{Condiciones ambientales}

El control ambiental del proceso se aplica tanto para la producción como para la medición. Durante la medición se debe controlar el ambiente, porque cuando se habla de alta precisión (por ejemplo, de $\left.|\times| 0^{-4}\right)$ las dimensiones son sensibles a cambios. $\mathrm{Si}$ las piezas se almacenan en un ambiente caliente podrían también alterarse sus dimensiones. También la alta precisión de los equipos de medición para estos casos hace que requieran en su mayoría de un ambiente controlado. En la investigación se encontró bastante control de este aspecto en la práctica, sin embargo, por su impacto es importante recalcar la necesidad de controlar tanto el ambiente de trabajo como el de la medición.

\section{Manipulación de las partes}

Existe gran sensibilidad en la manipulación de las partes cuando sus dimensiones meta son de alta precisión, al extremo de que debe cuidarse incluso la sujeción de las piezas y evitar su caída. En los casos que implican una serie de procesos adicionales al maquinado, por ejemplo, procesos de soldadura, la posibilidad de sufrir una alteración o incluso desprendimientos es mayor (un caso patente de la necesidad de cuidar la manipulación la presentan los moldes).

\section{Tamaño de las partes}

En el caso de las partes de grandes dimensiones, el maquinado es más complejo; un caso pueden representarlo incluso algunos moldes para inyección de plásticos.

En estos casos se puede requerir extremar el cuidado en la manipulación y transporte de las piezas, a pesar de que en las de gran tamaño las precisiones no son tan altas. Usualmente su geometría es compleja o con partes soldadas y por eso se deben manipular con cuidado.

\section{Procesos posteriores}

Los procesos posteriores a los que se someterá la parte deben ser considerados desde el diseño. Por ejemplo, un molde que se usará en un proceso de inyección de plástico, si su geometría es compleja y contiene partes soldadas, su uso posterior a altas temperaturas deteriorará eventualmente las juntas de soldadura.

\section{Material}

Este factor, quizás elemental, es conveniente detaIlarlo. El acero se encuentra en una gran gama de posibles aleaciones, de manera que es importante reconocer el material con el que se trabajará al establecer la dimensión objetivo para la pieza, pues en algunos materiales se podrá trabajar con valores más precisos y en otros no tanto. En el caso de materiales como las resinas plásticas, también existen muchas posibles combinaciones, por lo que se debe revisar el material previamente a la manufactura. Además, el material incide en la selección de los equipos, maquinaria y herramientas por utilizar. Este factor puede ser externo cuando el cliente lo especifica o interno cuando está sujeto a la decisión del fabricante.

\section{Filosofía de calidad}

La filosofía de calidad se incluye porque define las características del producto final. Cuando se tiene una filosofía de calidad de cumplimiento del objetivo se buscará lograr la dimensión meta y no limitarse a estar dentro de los límites permitidos. Una búsqueda de cumplimiento con lo especificado es fundamental para un maquinado preciso. Sin embargo, la filosofía de calidad conlleva un costo de la calidad.

Actualmente, la tendencia en la filosofía de calidad consiste en la excelencia. Una filosofía de excelencia 
conlleva un incremento en el costo de la manufactura, pero permite una reducción en los reclamos por el incumplimiento de los márgenes de ajuste. Los costos de reparaciones o reposiciones se han incrementado debido a políticas más exigentes de servicio al cliente y al aumento de las demandas legales por productos que incumplen las garantías ofrecidas por el fabricante, por lo que sobresale la importancia de una manufactura más precisa.

\section{Modelo para la manufactura de partes de acoples}

Para la mejora de las prácticas de manufactura conviene integrar los factores que se identificaron como importantes para la manufactura de partes de acoples o ensambles, en un modelo apropiado. En la figura I se esquematizan estos requisitos categorizados como aspectos internos, que obedecen a decisiones internas que se deben controlar; los aspectos externos corresponden a decisiones externas que no se pueden controlar pero que no se deben perder de vista pues los aspectos internos tienden a oscurecerlos. También se incluyen aspectos conexos, como el material, y aspectos tecnológicos, como la maquinaria y equipos, que ciertamente son de carácter interno pero que también obedecen a la tecnología disponible en el mercado.
Los aspectos internos se colocan en una segunda fase porque son aspectos sobre los que se tiene control. Precisamente en estos aspectos internos radica la mejora de las prácticas de manufactura mediante la búsqueda del balance de aspectos de la geometría de las partes, las tolerancias requeridas, el tamaño de los lotes de producción y los costos.

\section{Prueba del modelo para la manufactura de partes de acoples}

El modelo o guía se probó en un caso experimental de un acople comúnmente conocido como del pin que se inserta en un agujero (shaft and hole). El acople se muestra en la figura 2.

Para esta prueba muchos de los factores se darán por satisfechos sin mayor discusión, para poder valorar en mayor detalle el impacto de las tolerancias sobre la precisión de las dimensiones.

\section{Aspectos externos:}

- Ajuste final: Del estudio funcional se determinó una holgura "clearance" necesaria entre el pin y el agujero de 0,04mm.

- Tamaño de las partes: En unas piezas de $100 \mathrm{~mm}$ se hace un agujero, según lo indicado en el plano, de $10,04 \mathrm{~mm}$, en el que se inserta un pin de $10 \mathrm{~mm}$.

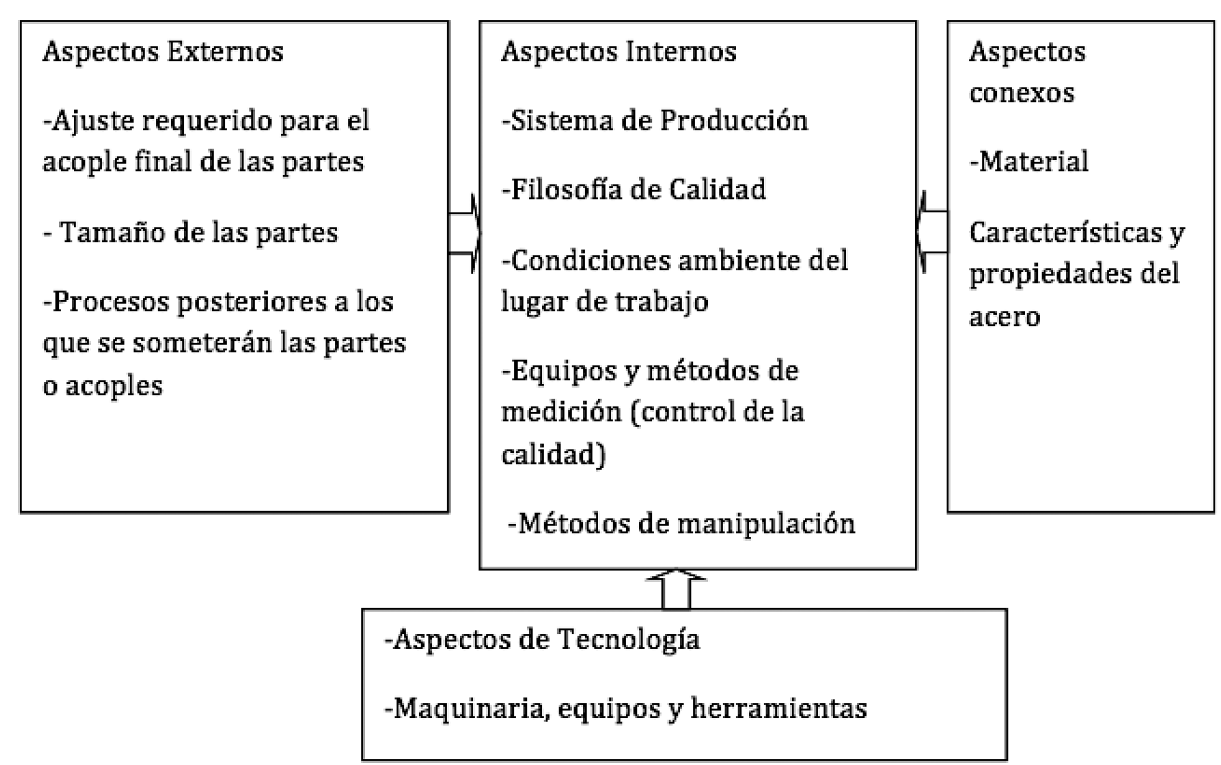

Figura I. Modelo para mejorar las prácticas de manufactura de partes de acoples. 


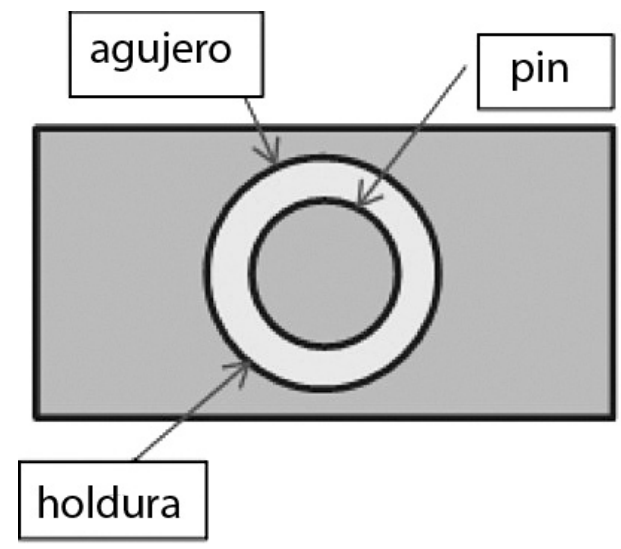

Figura 2. Caso del pin que se inserta en un agujero (vista superior).

- Procesos posteriores: Las partes no se someterán a procesos posteriores y en su uso no se expondrán a temperaturas extremas ni a golpes.

\section{Aspectos conexos:}

Material por procesar: Fue un acero $\bigcirc \mathrm{I}$ para fabricar las partes, por ser familiar para el operador de la maquinaria y adecuado para la herramienta disponible.

\section{Aspectos de tecnología:}

Equipos y herramientas: Se utilizó una fresadora CNC y una herramienta "fresa" de $5 \mathrm{~mm}$.

\section{Aspectos internos:}

- Manipulación de las partes: Para este caso las dimensiones y geometría de las partes, así como el bajo volumen de producción, permitieron una simple manipulación.

- Condiciones del ambiente: Las condiciones ambientales se mantuvieron a una temperatura de entre 20 y $25^{\circ} \mathrm{C}$, la cual no afecta el nivel de precisión alcanzada en las partes.

- Equipo y método de medición. Se utilizó un vernier digital; sin embargo, es conveniente usar un equipo de mayor capacidad. Para la medición se controló la forma de sujeción de la pieza y la temperatura ambiente.

- Filosofía de calidad: Para evidenciar el impacto de la filosofía aplicada se simularon dos escenarios, uno bajo una filosofía de alto cumplimiento con el objetivo y otro que se limita a estar dentro de los límites permitidos de tolerancia (Filosofía Cero Defectos).

Supóngase que los pines eran calibrados a $10 \mathrm{~mm}$, obligando al agujero a ser de 10,04 mm, de lo contrario el espacio libre no sería de $0,04 \mathrm{~mm}$. Por tanto, la especificación para el agujero sería de 10,04 -0 (para impedir diámetros inferiores a 10,04 mm). Sin embargo, para la manufactura conviene establecer un valor de tolerancia, pues de lo contrario una cantidad del producto estará fuera de especificación y se rechazará. El rango de la tolerancia de la máquina fresadora que hará el agujero puede usarse de referencia. Sin embargo, la filosofía de calidad que se siga influye bastante. A continuación se detalla el procedimiento para la designación del valor meta para la manufactura según estas dos filosofías de calidad. Para el ejemplo, se consideró inicialmente un rango de la tolerancia de $0,05 \mathrm{~mm}$ para la fresadora.

\section{- Prueba I: Filosofía de calidad: Cero Defectos}

Con una tolerancia unilateral de la máquina de $(+0,05-0)$ se establecería como valor máximo para la dimensión 10,09 y como límite mínimo 10,04. Si se usan estos valores como si fueran límites permisible para la dimensión, buscando "Cero Defectos" el valor meta por maquinar como dimensión para el agujero sería $(10,09+10,04) / 2=10,065$.

En un proceso bajo control, los diámetros de los agujeros presentarán un comportamiento de distribución de probabilidad normal (Teorema del Límite Central), con media $\mu$ y desviación estándar $\sigma$. Partiendo de la afirmación anterior se puede esperar que aproximadamente un 99,73 de los diámetros estará dentro del intervalo $\mu \pm 3 \sigma$.

\section{- Resultados esperados}

Con el pin de $10 \mathrm{~mm}$ y el agujero manufacturado a 10,065 , el espacio libre promedio será de 10,065$10,00=0,065 \mathrm{~mm}$. Para un proceso estable y en control, la desviación de los diámetros de los agujeros se puede obtener del rango de tolerancia de la máquina. Asumiendo el proceso de producción de la máquina bajo control se tiene: $6 \sigma=0.05$, por lo que $\sigma=0,0083$.

\section{- Prueba 2: Filosofía de Calidad: En el objetivo (On Target)}

Si se utiliza la misma máquina pero se acerca el valor meta por maquinar para la dimensión al objetivo 
de 10,04, por ejemplo a 10,05, entonces el espacio libre sería de 10,05-10,00=0,05mm, más cercano al buscado de 0,04.

\section{-Resultados esperados}

La desviación de los diámetros de los agujeros se mantendrá como $6 \sigma=0.05$, que resulta en $\sigma=0,0083$. Para este nuevo caso, la probabilidad de tener agujeros con diámetros inferiores a 10,04 sería:

$Z=(\mid 0,04-10,05) / 0.0083=0,01 / 0,0083=-1,2$ por lo que:

$P(X<10,04)=0,1150$

La figura 3 denota que si bien se acercó el valor meta de holgura "clearance" al especificado, ahora aproximadamente un $12 \%$ de las partes no tendrán la holgura requerida sino una donde el pin no podrá entrar. Lo anterior demuestra que es necesario reducir la desviación para lograr aproximarse al valor meta.

Otra forma de reducir la variabilidad sería mediante la mejora de los procesos de la maquinaria o bien cambiando a maquinaria más precisa, por ejemplo considere un centro de maquinado CNC con una fresadora de mayor precisión, que tenga un rango de tolerancia de $0,01 \mathrm{~mm}$.
-Prueba 3: Filosofía de Calidad:

En el objetivo (On Target)

Nuevamente, asumiendo esta tolerancia unilateral $(+0,0$ I - 0) el límite máximo podría ser 10,05 y el límite mínimo 10,04, el valor meta para la dimensión del agujero sería entonces $(10,05+10,04) / 2=$ 10,045 mm.

\section{-Resultados esperados}

El espacio libre promedio resultará en 10,045$10,00=0,045 \mathrm{~mm}$. Con la producción de la máquina bajo control se tiene: $6 \sigma=0.01$ por lo que $\sigma=0,00$ I 67 , que es una desviación menor.

\section{Discusión y conclusiones}

Como resultado de la investigación de casos y literatura consultada, se estimó necesario tomar en cuenta en la manufactura factores tales como: el ajuste requerido para el acople final, los equipos y herramientas disponibles para el maquinado, el sistema de producción, los equipos y métodos de medición, las condiciones ambientales durante el proceso, el tamaño de las partes y su manipulación, así como el material utilizado, los procesos o usos posteriores y la filosofía de calidad adoptada para la manufactura.

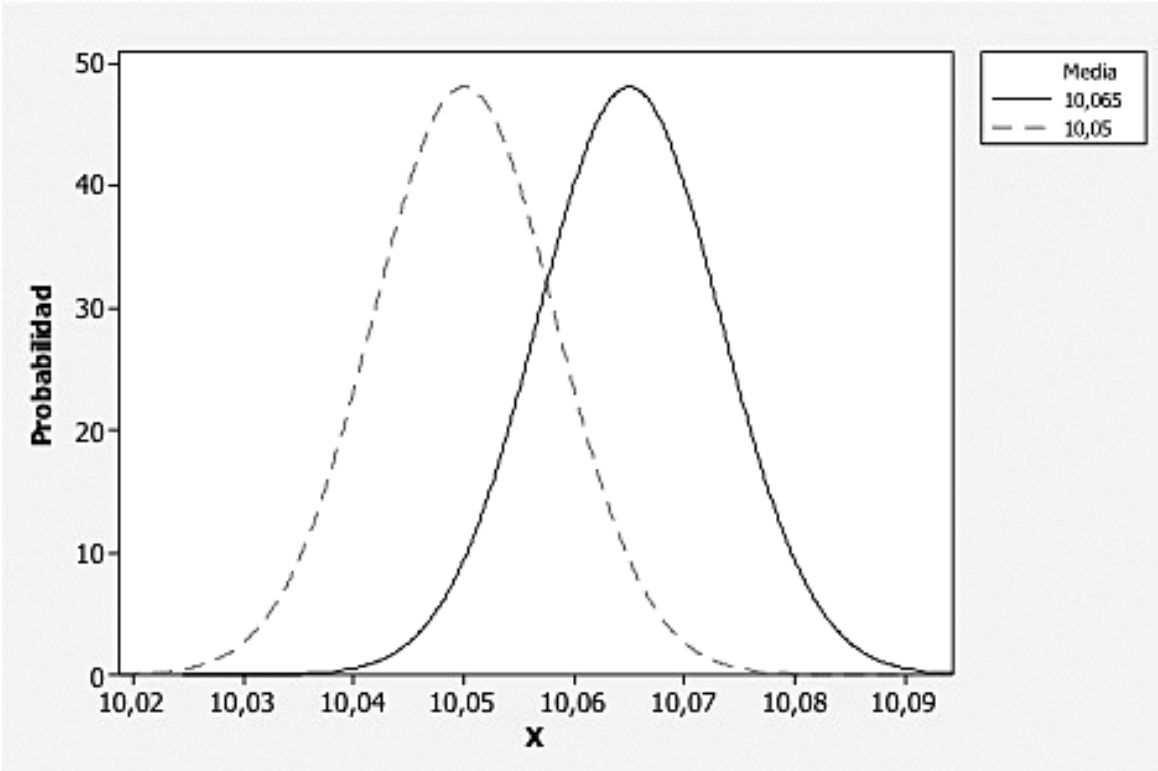

Figura 3. Distribución de diámetros de los agujeros (procesos controlados). 
Cuadro I. Comparación de las pruebas de asignación del valor meta por maquinar para el agujero con diámetro meta de $10,04 \mathrm{~mm}$.

\begin{tabular}{|c|c|c|c|c|}
\hline $\begin{array}{c}\text { Rango de tolerancia } \\
\text { de la máquina }\end{array}$ & $\begin{array}{c}\text { Filosofía de calidad para } \\
\text { determinar el valor por } \\
\text { maquinar }\end{array}$ & $\begin{array}{c}\text { Dimensión por } \\
\text { maquinar como meta }\end{array}$ & $\begin{array}{c}\text { Holgura } \\
\text { promedio } \\
\text { (respecto a I Omm) }\end{array}$ & $\begin{array}{c}\text { Probabilidad de un } \\
\text { espacio libre menor a } \\
0,04 \mathrm{~mm}\end{array}$ \\
\hline $0,05 \mathrm{~mm}$ & $\begin{array}{c}\text { Centrado entre límites } \\
\text { (Cero Defectos) }\end{array}$ & 10,065 & 0,065 & $<1 \%$ \\
\hline $0,05 \mathrm{~mm}$ & $\begin{array}{c}\text { En especificación meta } \\
\text { (En Objetivo) }\end{array}$ & 10,05 & 0,05 & $<1 \%$ \\
\hline $0,01 \mathrm{~mm}$ & $\begin{array}{c}\text { En especificación meta } \\
\text { (En Objetivo) }\end{array}$ & 10,045 & 0,045 & $<1 \%$ \\
\hline
\end{tabular}

Los lineamientos incorporados en el modelo para la mejora de las prácticas de manufactura denotan tener la fortaleza necesaria, porque permiten enfocar los aspectos que inciden con mayor impacto sobre los resultados del proceso. Ello amerita, por lo tanto, poner a prueba el modelo en casos que reflejen circunstancias cotidianas.

La filosofía de excelencia de la calidad conlleva un incremento en el costo de la manufactura, pero también una reducción en los reclamos por el incumplimiento de los márgenes de ajuste, por lo que constituye un factor importante del modelo.

La prueba experimental del modelo, por razones de tiempo y espacio, brinda poco detalle de la participación de los factores, pero demostró consistencia, y además permitió mostrar que cambiar el concepto de tolerancia por límites permitidos para la especificación perjudica el cumplimiento de los valores meta, aumentando la cantidad de producto desviado del objetivo. Dicha práctica además desperdicia la capacidad de exactitud de la maquinaria.

La prueba también demostró que la exactitud de la maquinaría que se utilice influye directamente en la consecución del valor dimensional meta.

\section{Bibliografía}

Al-Ansary, M.D. \& Deiab, I.M. (1997). Concurrent optimization of design and machining tolerances using the genetic algorithms method. International Journal of Machine Tools and Manufacture, Vol. 37, Issue 12, December 1997, pp. I721I73I, ISSN 0890-6955, 10.1016/S0890-6955(97)00033-3. Recuperado en febrero 2012 de: (http://www.sciencedirect. com/science/article/pii/S0890695597000333)
Bengtson, D. (2008) Develop a Process Control Plan. SME Manufacturing Engineering Vol. 141, September. Recuperado el 15 de abril 2011 de: http://www.sme.org/cgi-bin/findarticles.pl?\&08sem003\&ME\&2008090 I \&\&SME\&\#article

Bjorke, O. (1989) Computer-Aided Tolerancing. $2^{\text {nd }}$ edition. ASME Press.

Burton, T. (20/2). The generations of improvement. Industrial Engineer IIE, Vol. 44.

Creveling, C.M. (1997). Tolerance Design. A Handbook for Developing Optimal Specifications. Addison Wesley.

Fowlkes, W.Y. \& Creveling, C.M. (1995). Engineering Methods for Robust Product Design. Engineering Process Improvemente Series.

Guan, X., Wang, Y. \& Tao, L. (2009). Machining scheme selection of digital manufacturing based on genetic algorithm and AHP. Journal of intelligent manufacturing Vol. 20, N 6 . Recuperado el 20 de febrero del 2012 de Springer Link

Groover, M. (2007) Fundamentos de Manufactura Moderna: Materiales, Procesos y Sistemas. McGraw Hill.

Lin, S., Wang, H. \& Zhang, C. (1997). Optimal Tolerance Design for Integrated Design, Manufacturing and Inspection with Genetic Algorithms. Advanced Tolerancing Techniques 261281.

Madsen, D. (2009). Geometric Dimensioning and Tolerancing: based on ASME Y I 4.5-2009. Goodheart-Wilcox Co.

Maghsoodloo, S. \& Caleb Li, M. (2000). Optimal asymmetric tolerance design. IIETransactions 32: | | 27- | | 37. Recuperado en diciembre 201 I de Springer.

McMahon, C. \& Browne J. (1998). CAD CAM Principles, practice and manufacturing management. 2 ed. Addison Wesley Longman.

Mehta, M. (2009). Kick down the goalpost. Industrial Engineer IIE. Vol. 42. October.

Wortman, B. (2006). Certified Quality Engineer PRIMER. Quality Council of Indiana. 